\title{
HIGHER-RANK NUMERICAL RANGE IN INFINITE-DIMENSIONAL HILBERT SPACE
}

\author{
RUBÉN A. MARTÍNEZ-AVENDAÑO
}

Abstract. In this paper we calculate the higher-rank numerical range, as defined by Choi, Kribs and Życzkowski, of selfadjoint operators and of nonunitary isometries on infinite-dimensional Hilbert space.

Mathematics subject classification (2000): 47A12, 47B15, 15A60.

Key words and phrases: Higher-rank numerical range, selfadjoint operator, nonunitary isometry.

\section{REFERENCES}

[1] Man-Duen Choi, Michael Giesinger, John A. Holbrook and David W. Kribs, Geometry of higher-rank numerical ranges, Linear and Multilinear Algebra 56 (2008), 53-64.

[2] Man-Duen ChOI, John A. HolbrooK, David W. KRIBS, AND Karol ŻYCZKowski, Higher-rank numerical ranges of unitary and normal matrices, Operators and Matrices 1 (2007) 409-426.

[3] MAN-Duen ChOI, DAVID W. KRIBS AND KAROL ŻYCZKOWSKI, Higher-rank numerical ranges and compression problems, Linear Algebra Appl. 418 (2006) 828-839.

[4] John B. Conway, A Course in Functional Analysis, second edition, Springer, New York, 1990.

[5] DOUgLAS R. FARENICK, Matricial extensions of the numerical range: a brief survey, Linear Multilinear Algebra 34 (1993) 197-211.

[6] Hwa-Long Gau, Chi-Kwong Li and Pei Yuan Wu, Higher-rank numerical ranges and dilations, J. Operator Theory, to appear.

[7] PaUl R. Halmos, A Hilbert Space Problem Book, second edition, Springer, New York, 1982.

[8] ChI-Kwong Li, Yiu-Tung PoOn And Nung-Sing Sze, Condition for the higher rank numerical range to be non-empty, Linear and Multilinear Algebra, to appear.

[9] ChI-Kwong Li, YIU-Tung PoOn AND Nung-Sing SzE, Higher rank numerical ranges and low rank perturbations of quantum channels, preprint.

[10] CHI-KwOng Li AND Nung-Sing SZE, Canonical forms, higher rank numerical ranges, totally isomorphic subspaces and matrix equations, Proc. Amer. Math. Soc., to appear.

[11] ChI-KwOng Li AND NAM-KIU Tsing, On the $k$-th matrix numerical range, Linear Multilinear Algebra 28 (1991) 229-239.

[12] Rubén A. Martínez-Avendaño and Peter Rosenthal, An Introduction to Operators on the Hardy-Hilbert Space, Springer, New York, 2007.

[13] Peter Rosenthal and Heydar Radjavi, Invariant Subspaces, second edition, Dover, Mineola, NY, 2002.

[14] Béla SZ.-NAGY AND CipRIAn FoIAŞ, Harmonic Analysis of Operators in Hilbert Space, North-Holland, Amsterdam, 1970.

[15] J. WeRMER, On invariant subspaces of normal operators, Proc. Amer. Math. Soc. 3 (1952) 270-277.

[16] Hugo J. Woerdeman, The higher rank numerical range is convex, Linear Multilinear Algebra, 56 (2008) 65-67. 